\title{
Las glaciaciones de la Sierra Nevada de Santa Marta
}

\author{
Henry Cornelius Raasveldt
}

Rev. Acad. Colomb. Cienc. Ex. Fis. Nat. 1957, (9): 469-482.

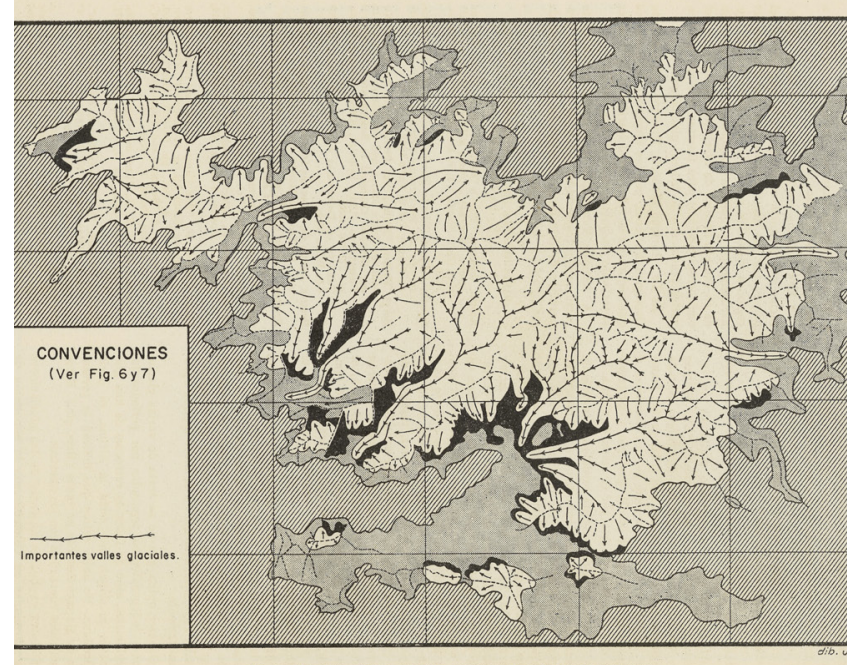

Extensión máxima del hielo durante el Gran Estado Mamancanaca

\section{Henry Cornelius Raasveldt (1913-1984)}

Nacido en Surabaya, Indonesia, fue un geólogo pionero en la práctica de la fotogeología en Colombia, que llegó al país en el año 1952 y quien con un equipo de geólogos de origen extranjero y nacional de variadas especialidades, convocado por el Servicio Geológico Nacional (SGNC), emprendiera un amplio estudio del territorio colombiano durante la segunda mitad del siglo XX.

Sus trabajos se enfocaron principalmente en la elaboración de extensos mapas fotogeológicos y geomorfológicos con especial énfasis en lagunas, humedales y glaciares.

Además de los estudios netamente geológicos, su trabajo se ocupó también de temas como los fundamentos de la visión estereoscópica y su utilidad en la fotointerpretación y de cómo esta última práctica hacía más accesible, económica y logísticamente, el estudio de amplias zonas del territorio nacional. También se preocupó por la unificación de los mapas producidos por el SGNC bajo un mismo sistema de coordenadas con origen en Bogotá. Raasveldt murió en Straubing, Alemania en 1984.
La Sierra Nevada de Santa Marta es una de las montañas más interesantes del planeta tanto por su historia geológica como por su biota. A pesar del gran interés que suscita, es aún muy poco lo que conocemos de ella. Por ejemplo, cuándo alcanzó su elevación actual es aún un gran misterio. Otro de las grandes preguntas a resolver es la historia de sus glaciares, especialmente ahora que están desapareciendo y algunos predicen que dejarán de existir para fines de siglo, lo cual podría traer grandes transformaciones a la dinámica hídrica de todo el nororiente de Colombia.

El artículo publicado por Raasveldt presenta una investigación pionera en la historia geológica de la Sierra Nevada y hoy en día, es aún un artículo de consulta obligatoria para cualquiera que pretenda estudiarla.

Raasveldt presenta el primer mapa que detalla las glaciaciones en la región usando la primera serie de fotografías aéreas de la Sierra tomadas por el Instituto Agustín Codazzi en la década de los 50. Era tal el desconocimiento de la topografía de la Sierra que Raasveldt tuvo que bautizar una gran cantidad de picos y cierros que se observaba en las fotos pero que aún no tenían nombres (ej Pico Tairona, Cerro Acosta). Raasveldt detalla las diferentes características glaciales que encuentra, y no se limita a describirlos, sino que propone hipótesis sobre su formación. El artículo finaliza postulando que existieron tres estados de glaciación que en su máxima extensión pudieron haber ocupado un 20\% del área total de la Sierra. Les recomiendo leer detenidamente este artículo el cual es un ejemplo de ciencia de alta calidad y demuestra como el conocimiento básico es esencial para el desarrollo de cualquier sociedad.

Carlos Jaramillo, Ph.D.

Miembro Correspondiente

Sebastián Gomez

Estudiante de pregrado Geología 


\title{
LAS GLACIACIONES DE LA SIERRA NEVADA DE SANTA MARTA
}

\author{
H. C. RAASVELDT
}

\section{Introducción.}

Varios pioneros de la geología de este país han publicado datos sobre los fenómenos de glaciación, como por ejemplo sobre morrenas, valles glaciales y bloques erráticos, observados a niveles mucho más bajos que las altitudes en que se encuentran actualmente la neviza y el hielo. Aunque no se sabe todavía con seguridad si en el territorio colombiano se presentaron los mismos periodos de intensa glaciación como los que se conocen ya bastante bien en el viejo mundo europeo o en el nuevo continente norte-americano, el material observado fue lo suficientemente claro para concluir que las glaciaciones colombianas fueron más extendidas de lo que se estaba inclinando a suponer con respecto a un país que, por su posición geográfica, es tropical.

Las antiguas glaciaciones de estas latitudes han sido presentadas muy sumariamente en las publicaciones conocidas y ningún geólogo o glaciólogo ha dado a conocer la intensidad y la extensión de los fenómenos de manera objetiva en un mapa glaciológico. Víctor Oppenheim quien estudió más en particular las glaciaciones de la Cordillera Oriental pudo distinguir varios períodos o estados de glaciación y presentó un croquis con algunas curvas de nivel que no caracterizan estos estados sino muy esquemáticamente, $\mathbf{y}$ así ya nos dio una idea, aunque muy superficial de los fenómenos.

$\mathrm{Su}$ croquis, sin embargo, no es un mapa glaciológico. La razón por la cual no se publicaron mapas mejores, que señalen la distribución de vestigios glaciales, es bastante obvia: no existían cartas básicas que representaran los accidentes topográficos con suficiente fidelidad para poder localizar las observaciones terrestres. La confección de un mapa glaciológico por levantamiento terrestre es un asunto demasiado dispendioso en tiempo y dinero para justificar su ejecución.

A esto se puede añadir que son escasos los centros habitados en las regiones altas del país o están industrialmente tan poco desarrollados que sus requerimientos en recursos naturales no son importantes, como para que se incluyan los depositos glaciales en la zona de interés económico, caso que sí ocurrió, por ejemplo, en ciertas zonas de los Estados Unidos.

En tales condiciones, el estudio glaciológico en Colombia tiene todavía un carácter puramente científico y esto explica que los fenómenos glaciales hayan sido enfocados muy pocas veces. La intensa actividad, que a instigación del Instituto Geográfico de Colombia "Agustín Codazzi", se está desarrollando para fotografiar la topografía del país desde el aire, ha abierto vastas perspectivas para la confección de cartas sobre fenómenos geológicos, con más eficacia y con mucho menor costo de lo que se puede obtener mediante mediciones a ras de la tierra.

Uno de los grandes complejos montañosos aero. fotografiados casi integramente es la Sierra $\mathrm{Ne}$ vada de Santa Marta. Para nosotros, que estamos encargados de definir las condiciones geológicas del país a base de las fotografías aéreas, no fue grande el esfuerzo adicional que se requirió para complementar el estudio rutinario y separar los fenómenos glaciales, hasta obtener un mapa especificamente glaciológico. En efecto gasté unos tres días, para alistar el borrador del mapa glaciológico presentado aquí. A continuación utilicé las noches de varias semanas para completarlo $\mathbf{y}$ para preparar el texto $\mathbf{y}$ atender a varios otros aspectos hasta terminar el presente estudio.

El estudio de los vestigios glaciales antiguos, testigos de una extensión del hielo mucho mayor que la actual, tiene un atractivo especial que distingue tal estudio de las investigaciones precuaternarias. Este interés tal vez lo suscita el hecho de que el hombre ya había aparecido en varias regiones de la superficie terrestre y porque sabemos que en estas partes su vida estaba íntimamente conectada con un ambiente de hielos y nevizas. Además, el evocar en la mente los casquetes de hielo que cubrían inmensas áreas de un país situado en el cinturón tropical de la tierra, como es el caso de Colombia, no puede sino llenar la imaginación con paisajes grandiosos, despertar la curiosidad científica, e instigar la investigación.

Para iniciar un estudio glaciológico más detenido, la Sierra Nevada de Santa Marta ofrece dos ventajas que no se encuentran en otras regiones y que son las siguientes:

1. La Sierra Nevada, separada de las otras cordilleras por bajos planos aluviales, forma en sí misma una unidad orográfica, y la región de glaciaciones, que tiene sus límites naturales, no es muy vasta. Esto es un factor favorable para iniciar un primer mapa glaciológico.

2. La cresta nevada de la Sierra presenta una buena base para correlacionar las distintas glaciaciones, porque las podemos enumerar de arriba hacia abajo contando desde la actual hacia atrás en el tiempo. También esto es una gran ventaja, porque muy poco se sabe actualmente de las glaciaciones del país, es decir, ni de sus dimensiones, 
diseminación y de su periodicidad, ni de la morfología de los restos glaciales.

Después de haber terminado el mapa inclú toda la nomenclatura conocida de la región. Para esto consulté el excelente mapa hecho por la expedición Cabot y publicado por el Instituto Geográfico con sede en Nueva York.

Este es el único mapa fidedigno que sirve para tales fines. Los demás existentes son tan generalizados que no tienen nada en común con las condiciones topográficas verdaderas. Para evitar el empleo de nombres que no corresponden a la realidad, decidí no utilizar tales mapas defectuosos. Toda la nomenclatura, como también las altitudes de todos los picos y cerros que se encuentran en el mapa de Cabot, fueron incorporadas en el presente mapa glaciológico. Otra importante fuente para completar la nomenclatura la encontré en el alpinista Erwin Kraus quien visitó la Sierra Nevada rarias veces. Es probablemente el mejor conocedor de esta región, actualmente presente en el país, así que le fue posible completar el mapa con los siguientes nombres: los ríos Donachuí (d 6) ${ }^{1}$, Tambor (d 6), Guatapurí (c 6), San Miguel ( a 6), Cuturataca ( e 4), Aduriameina (e 4), Mamancanaca ( d 3, d 4 ), Cataca (c 4), Palomino ( a 4 ), Aracataca ( d 2), y Tucurinca ( c 2); los caseríos o grupos de chozas: Donachuí (d 6), Sogrome (d 6), Secaracungüe ( e 6), Kancurnaca ( c6), Meollaca ( c 6), el paso de Bellavista ( d 4) y los lagos del Mamo y Naboba ( c 4 ).

Algunos errores del Mapa Cabot fueron corregidos, como, por ejemplo, la posición del río Guatapurí. Según E. Kraus, el río Aduriameina es afluente del río Mamancanaca. Donde éste se une con el río Cataca, que drena los grandes lagos, empieza a formarse el río Aracataca que entra por fin en la Ciénaga Grande de Santa Marta.

Aún con las notables adiciones de E. Kraus la nomenclatura es todavía demasiado esparcida, lo que dificulta apreciablemente la discripción del contenido del mapa por falta de puntos de referencia geográfica. Por esta razón me permití aplicar provisionalmente a varios picos y cerros los nombres de científicos y alpinistas, también de funcionarios del Instituto Geográfico de Colombia, los cuales en muchas ocasiones me prestaron su generosa cooperación. Estos nombres, que sa han pnesto con letras inclinadas en el mapa, se encuentran reunidos en la siguiente lista:

Cerro Hettner (a2) por Alfred Hettner, uno de los pioneros de la geología en Colombia, quien contribuyó al conocimiento de la Sierra Nevada.

Cerro Garavito (b2), por Julio Garavito, científico de renombre en cartografía, magnetismo terrestre y meteorología.

1 La letra $y$ la cifra indican el cuadrado de ln euadrícula de 10 en 10 kilometros donde se encuentra el detalle en el mapa glaciológico: ver los márgenes del mapa.
Cerro Darío Rozo (b2), por el ingemiero Darío Rozo M., muy conocido por sus estudios geográficos.

Cerro Gansser (a3), por August Gansser, el conocido geólogo strizo, quien tenía una grande pasión por la Sierra Nevada y andazmente la escaló solo.

Nevado Parra Lleras (b3), por Ernesto Parra Lleras, jefe de la Sección de Aerofotogrametría, a quien se suele llamar "el alma del Instituto Geográfico".

Pico Menders (b3), por Federico Guillermo Mendershausen, del Instituto Geográfico, fundador de la aerofotogrametría en Colombia a quien sus colaboradores, con un criterio práctico y a la vez con cariño, apodan "Menders".

Pico Ruiz Erazo (b3), por José Ignacio Ruiz Erazo, el estimado ingeniero $y$ querido Director del Instituto Geográfico.

Cerro Karsten (c3), por Hermann Karsten, otro pionero alemán en la geología de Colombia, cuando la República todavía se llamaba Nue. va Granada.

Cerro Marmillod (c3), por F. Marmillod, conocido alpinista que escaló también la Sierra Nevada de Santa Marta, acompañado de su esposa.

Cerro Arias (d3), por Hernán Arias, empleado de la Sección de Aerofotogrametría, quien parece conocer todas las 120.000 fotografías del Instituto Geográfico.

Cerro Acosta (d3), por Joaquín Acosta, destacado geógrafo quien hace más de un siglo efectuó una contribución a la geología de la Sierra Nevada, llamada por él Sierra Tairona.

Pico Ruiz Wilches (b4), por Belisario Ruiz Wilches, el Néstor de los científicos colombianos.

Pico Vespucio (c4), por Américo Tespucio, quien $n o$ debe estar lejos de Cristóbal Colón (en b4 abreviado C. C.), y quien ha entrado de nuevo en la actualidad por la elogiada biografía de Germán Arciniegas.

Pico Tairona (c4), por la extinguida nación inds. gena de los Taironas, cuyo nombre se ha usado para designar la Sierra Nevada de Santa Marta (ver Acosta y E. Hubach en la bibliografía).

Cerro Pérez Arbeláez (d4), por el R. P. Enrique Pérez Arbeláez, el conocido fisiógrafo-botánico cuyas importantes publicaciones constituyen una lectura agradable por la claridad y el espíritu crítico y a veces humorístico en que están escritas.

Curo Kraus (d4), por Erwin Kraus, el joyero, quien además se destaca con "hobbies" muy cultivados como el alpinismo y la fotografía (ver figuras 9-19), probablemente el mejor conocedor de la Sierra Nevada. 
Cerro Stutzer (e4), por Otto Stutzer, el geólogo alemán cuyos numerosos estudios son fundamentales para el país y quien reconoció los vestigios glaciológicos de la Cordillera Oriental.

Cerro Grosse (e4), por el destacado geólogo alemán Emil Grosse, cuyos estudios y detallados mapas de la región carbonífera de Antioquia son ejemplares.

Cerro Guhl (a5), por Ernesto Guhl, actualmente jefe del Depto. Técnico de la Seguridad Social Campesina, el geógrafo que se erigió un monumento con su estudio sobre el Departamento de Caldas, además conocedor y autor de un artículo sobre la Sierra Nevada.

Cerṛo Grière (b5), por Raymond Grière, "ataché" de la legación francesa, entusiasta alpinista, quien publicó un interesante artículo sobre la historia de las conocidas ascenciones a la Sierra Nevada.

Pico Tulio Ospina (b5), por Tulio Ospina Vásquez, fundador y primer rector de la Escuela de Minas de Medellín, cuyos conceptos geológicos han sido muy apreciados.

Pico Corlazzi (c5), por el destacado geógrafo Agustín Codazzi, cuyo nombre se confirió a una población y a un importante instituto.

Nevado Ramírez (c5), por el R. P. Jesús Emilio Ramírez, S. J., el geofísico con un extraordinario "pushing power", máximo promotor de los estudios telúricos en Colombia.

Cerro Laverde (c5), por el coronel Luis Laverde G., subdirector del Instituto Geográfico, quien encabeza la avanzada militar que explora exitosamente el terreno de la fotogrametría.

Cerro Reclus (d5), por Jean Jacques Elisée Reclus (1830-1905), el renombriado geógrafo francés, autor de la extensa obra "Geógraphie Universelle" quien visitó la Sierra Nevada y trato de establecer una colonia agrícola en sus flancos.

Cerro Mora (d5), por Marcos Mora Chacón, jefe de la Sección de Geodesia del Instituto Geográfico, cuya labor es base esencial para la cartografía del pais.

Cerro Cabot (d5), por Thomas D. Cabot, quien encabezó una expedición que por vías aéreas y terrestres produjo el primer mapa fotogramétrico de una gran parte de la Sierra Nevada.

Cerro Arjona (d5), por Belisario Arjona E., jefe de la Sección de Recopilación del Instituto Geográfico, quien elaboró el sistema de proyección actualmnte en uso para las planchas topográficas de Colombia.

Cerro Cuenet (e5), por el topógrafo-geodesta suizo G. H. Cuenet, apasionado alpinista que escaló varios picos de la Sierra Nevada.
Cero Valencia (b6), por Luis Felipe Valencia Lozano.

Cuchilla Rodríguez (c6), por Jorge Noel Rodríguez. Los dos últimos son fotogrametristas del Ins. tituto Gográfico, quienes con expertas manos y pies "manejan" complicadísimos instrumentos y en un minúsculo globito dirigible viajan por todo el país, produciendo así las hermosas cartas topográficas.

Cerro Royo y Gómez (d6), por el activo geólogo-paleontólogo español José Royo y Gómez, quien vinculó definitivamente su nombre con la investigación geológica de este país.

El presente mapa glaciológico fue construído con base en cartones ranurados (slotted templet layout) armados sobre los puntos geodésicos disponibles en los flancos occidental y meridional de la Sierra Nevada. No existía ningún fidedigno control terrestre en el flanco septentrional y además había una faja de terreno no fotografiado inmediatamente al borde oriental del mapa. En estas condiciones el mapa no puede ser preciso y la posición de los detalles debe ser muy aproximada. El método de los cartones ranurados, por lo demás, no presenta los mejores resultados en un terreno que tan bruscamente se eleva desde el nivel del mar hasta a más de $5.700 \mathrm{mts}$. Las coordenadas expresadas en kilómetros, que se encuentran en los márgenes del mapa, corresponden a las planas del Instituto Geográfico con base de $\mathrm{X}=1.000 \mathrm{~km}, \mathrm{y} \quad \mathrm{Y}=1.000$ km para Bogotá.

\section{Localización y delimitación del área con ves- tigios de glaciaciones.}

La Sierra Nevada de Santa Marta es, a grandes rasgos, wi gigantesco tetraedro que se compone casi completamente de rocas precretáceas. El Cretáceo sólo aflora en dos esquinas de su base triangular: en la punta nordeste y en la del sur (fig. 1) ${ }^{2}$. Por lo demás, está rodeada del Cuaternario mientras que la punta nor-occidental la baña el Mar Caribe. Una estrecha divisoria formada de sedimentos cretáceos conecta la esquina nordeste del triángulo con los Montes de Oca, un ramal de la Serranía de Perijá situado en el límite colombo-venezolano. Esta divisoria es el confín NE de la cuenca del río Cesar. El extremo septentrional de esta cuenca, por un extraño capricho de la naturaleza, lo desagua el río Ranchería que se abre paso hacia el norte por un estrecho cañón cortado por él en las calizas cretáceas. Sobre los dos afloramientos mencionados del Cretáceo reposa el Terciario que lleva varios mantos de carbón en la parte superior de la cuenca del Cesar donde se encuentran las minas del Cerrejón.

\footnotetext{
2 En la figura 1 no hemos tomado en enenta algunos afloramientos del Cretáceo al norte del Macizo de Santa Marta. Estos afloramientos no son autóctonos $y$ deben conectarse con desplazamientos horizontales a lo largo de fallas de rumbo secundarias $y$ paralelas a la importante falla de Oca. Ver también
} nota 3. 


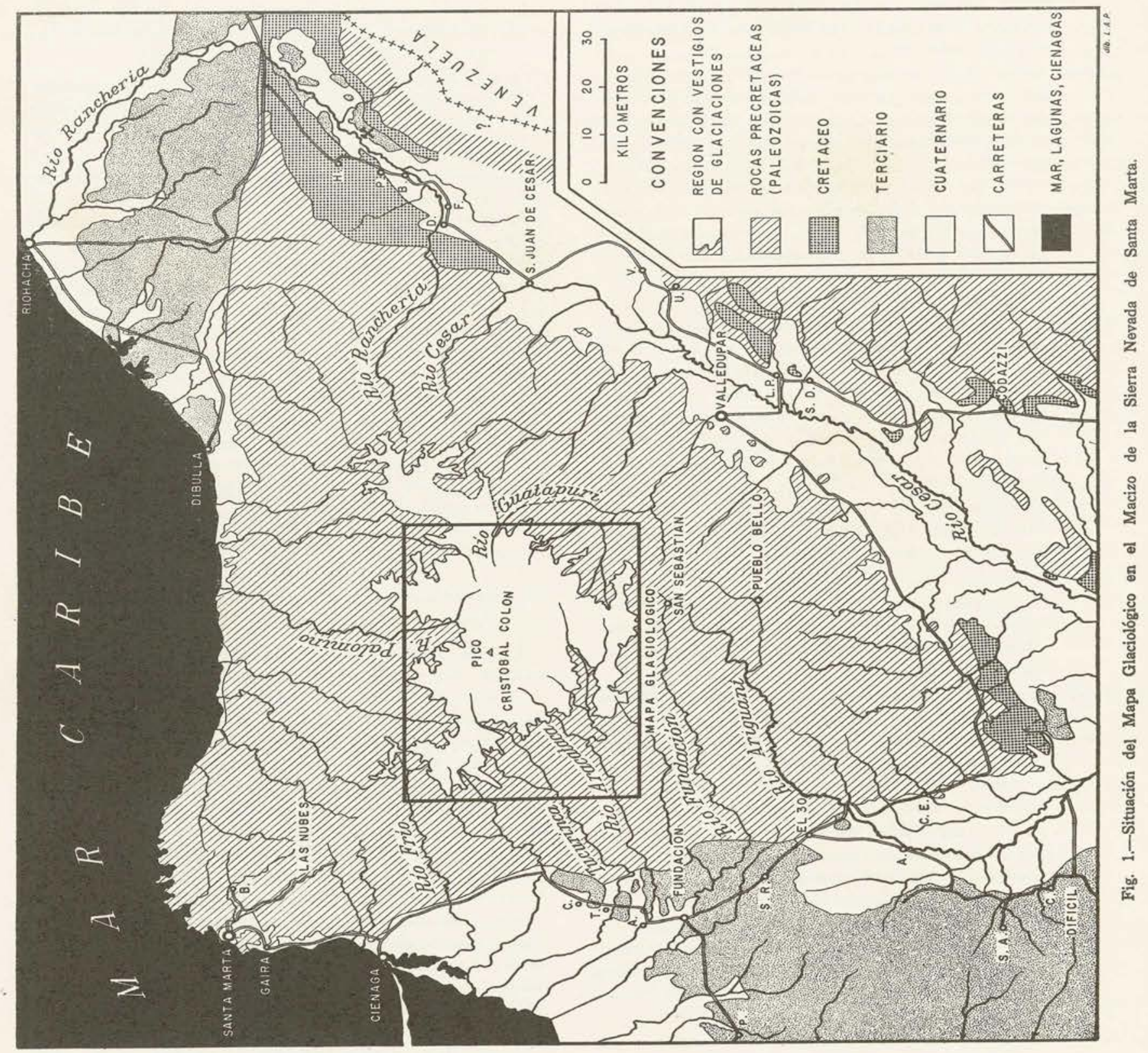


El Terciario que aflora a lo largo del costado septentrional del triángulo base da origen a un terreno ligeramente accidentado; pero hacia el nordeste la formacion desaparece debajo del gran plano aluvial cuaternario que principia cerca de Riohacha $\mathbf{y}$ se extiende hasta la península de La Guajira y la costa venezolana.

En el costado occidental del triángulo, inmediatamente al E y SE de Fundación, se encuentran unos afloramientos del Terciario de reducida extensión. Todos estos terrenos terciarios son bajos y sobresalen sólo varias decenas de metros de los planos aluviales que completan la delimitación de la base triangular de la Sierra Nevada. Estos planos coresponden a los xíos Cesar y Ariguaní en el sureste, y suroeste, con una llanura al NW de Fundación que se extiende hasta la vasta laguna conocida bajo el nombre de Ciénaga Grande de Santa Marta.

De ahí se infiere que la Siera Nevada de Santa Marta es una unidad orográfica independiente, circunstancia especialmente notable porque a base de afinidades litológicas y petrográficas se le considera generalmente como continuación de la Cordillera Central. Expliqué recientemente esta peculiaridad mediante la hipótesis de la "Gran Falla" a lo largo de la cual el macizo de Santa Marta se ha desplazado horizontalmente más de 120 kilómetros hacia el norte ${ }^{3}$. Adicionalmente el macizo debe haberse levantado varios miles de metros a lo largo de la misma falla.

La Sierra Nevada de Santa Marta es pues, una unidad orográfica muy especial y su abrupta elevación a una altura de más de 5.700 metros sobre una distancia horizontal del mar de apenas 48 kilometros es única en el mundo.

Es obvio que en estas condiciones debe presentarse una erosión extremadamente fuerte y ello es la causa de haberse preservado sólo una pequeña parte de las antiguas glaciaciones pleistocenas (cuaternarias). Este hecho lo demusstra claramente el ad. junto mapa glaciológico en el cual la línea roja indica hasta qué altura ha progresado la erosión. Está bien destacada la condición de cómo varias formas glaciológicas terminan abruptamente en esta línea, por ejemplo la topografía glacial del flanco S y SW del Cerro Arias (d3), la de la vertiente meridional de los cerros Stutzer y Grosse (e4), como también la de los cerros Arjona, Mora (d5), Royo y Gómez (d6), hacia el SE.

La mencionada línea es solamente un símbolo esquemático; en realidad se trata de una zona de tran. sición, más o menos vaga, en la cual la labor destructora de la erosión disminuye gradualmente hacia arriba. Esta zona no se encuentra siempre a la misma altura y puede fluctuar entre los 2.800 y 3.300 metros. En algunas partes, ella es bastante

3 "Fallas de rumbo en el Nordeste de Colombia". Revista del Petroleo No 64, mayo 1956. estrecha y por lo tanto está bien definida. En otros lugares es ancha y en estos casos no hay criterios claros para trazar la línea de una manera precisa.

La línea de erosión está indicada en la figura 1 en relación con el marco del mapa glaciológico. El lector podrá observar que el mapa no abarca toda la zona con vestigios de glaciaciones, pero sí el sector más importante.

\section{Los glaciares actuales.}

Los glaciares actuales se concentran alrededor de las crestas más altas que pueden subdividirse en las siguientes unidades.

1. El Nevado Parra Lleras con dos glaciares en el flanco septentrional y nevizas no perennes en la vertiente meridional.

2. Las crestas nevadas entre los Picos Menders. Ruiz Wilches.

3. El gran complejo de nevados con los picos RuizErazo, Simons, S. Bolívar, Cristóbal Colón y A. Vespucio. Los dos picos C. Colón y S. Bolívar son los más altos de la Sierra Nevada de Santa Marta, ambos con 5.775 metros de altura según el mapa de Cabot.

4. El complejo gemelo que se compone de dos sierras: la de los picos Ojeda-Codazzi-Tulio Ospina y la otra con los picos La Reina y Nevado Ramírez.

5. La sierra nevada con los picos Tairona-El Guardián.

Los glaciares miden rara vez más de dos kilómetros de largo. Predominan los tipos glaciares de pendientes, casquetes de hielo inclinados, que están situados en una sola vertiente del nevado, separados de las otras por crestas divisorias puntiagudas. A veces terminan en lenguas glaciales no muy desarrolladas, pero no se presentan glaciares de valle. Para que tales tipos de glaciares puedan formarse, es necesaria la presencia de masas de hielo mucho más grandes: Las terminaciones de 10 slaciares o de las lenguas no avanzan por lo general abajo de los 4.700 metros; en un caso, una lengua más larga que las otras llega a casi 4.500 metros.

Muchos glaciares están atravesados por nume. rosas rupturas perpendiculares a la dirección de la corriente de hielo. Esto indica un estiramiento de los glaciares en la dirección del hilo de la corriente del hielo, fenómeno que sugiere que está disminuyendo la caída de nieve. Por consiguiente no habrá suficiente aporte de neviza y hielo en la parte superior de los glaciares para compensar por completo el material que desciende por la gravedad a lo largo de las fuertes pendientes. Por lo tanto es probable, juzgando sólo a base de la morfología de los glaciares mismos, que ellos estén retrocediendo. Hay indicios, explicados a continuación, de que el retroceso se ha iniciado ya hace algún tiempo. 


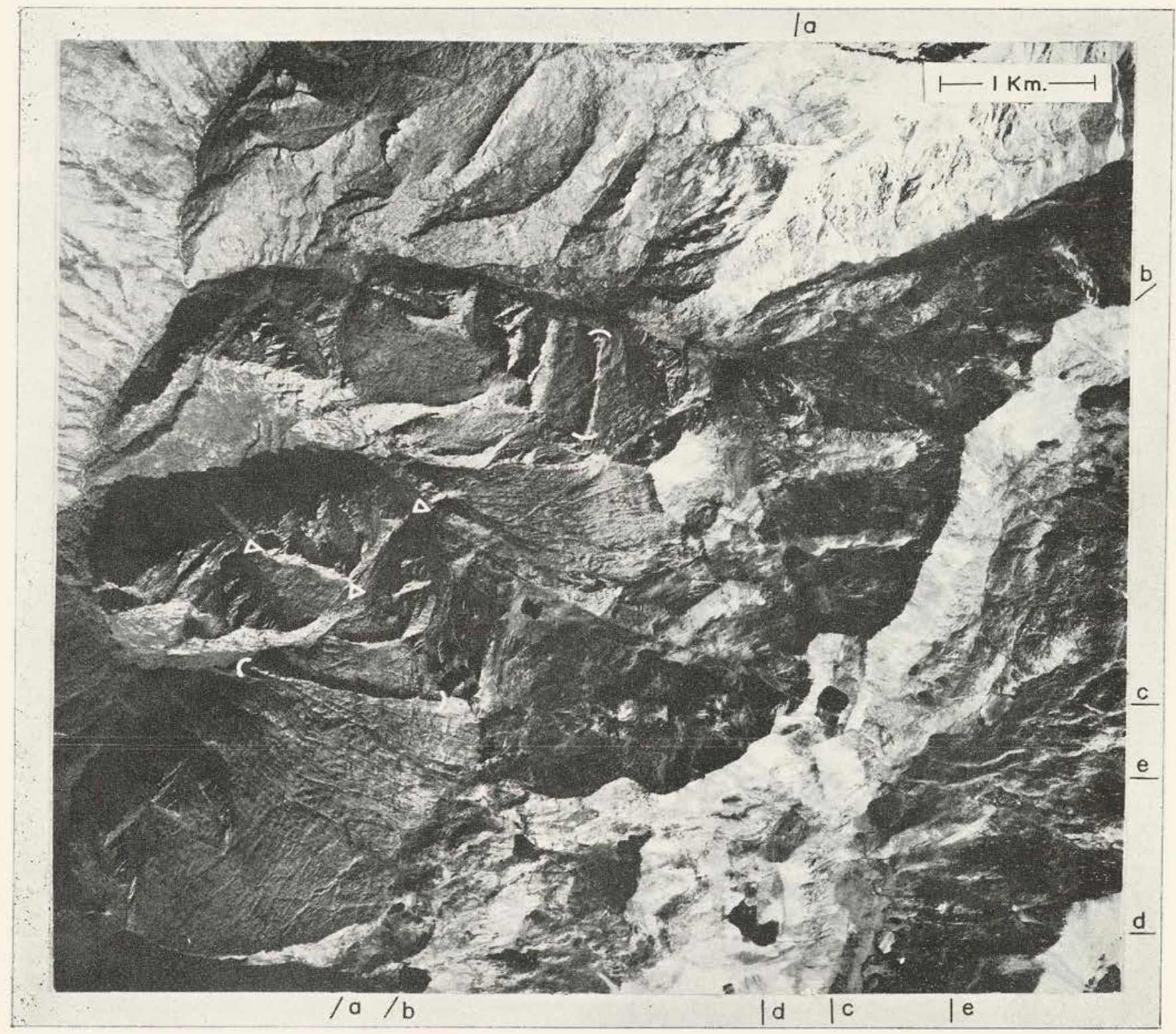

Fig. 2.-La región al ceste de las flechas a-a muestra una topografía de planos cepillados por la acción erosiva de los casquetes de hielo que antiguamente cubrian la región. En esta área el movimiento del hielo fue en general de ESE a WNW. La zona entre a-a y b-b ya representa la transición entre la topografía de planos cepillados y la de las ollas glaciales, tipo de erosión que domina completamente en la zona al este de b-b. Los depósitos glaciales aumentan desde a-a hacia el este mientras que la roca al oeste de a-a está desnuda y desprovista de cantidades apreciables de material glacial. En la última zona son conspícuas las numerosas costillas (en la foto, dos de éllas entre paréntesis blancos) que subdividian el antiguo casquete glacial en muchas unidades. Grupos de varias costillas pueden formar una unidad triangular más grande (entre los 3 triángulos en la foto).

Los laguitos al este de a-a están generalmente formados por la acción del bloqueo que efectúan las pequeñas morrenas frontales, como por ejemplo los laguitos c-c y d-d. Dos morrenas son bien visibles en la intersección de las flechas e-e. A la derecha de b-b el hielo bajó del NNE al SSW.

Es una fotografía aérea vertical del Instituto Geográfico Agustín Codazzi, como lo son también las figuras 3 y 5. 


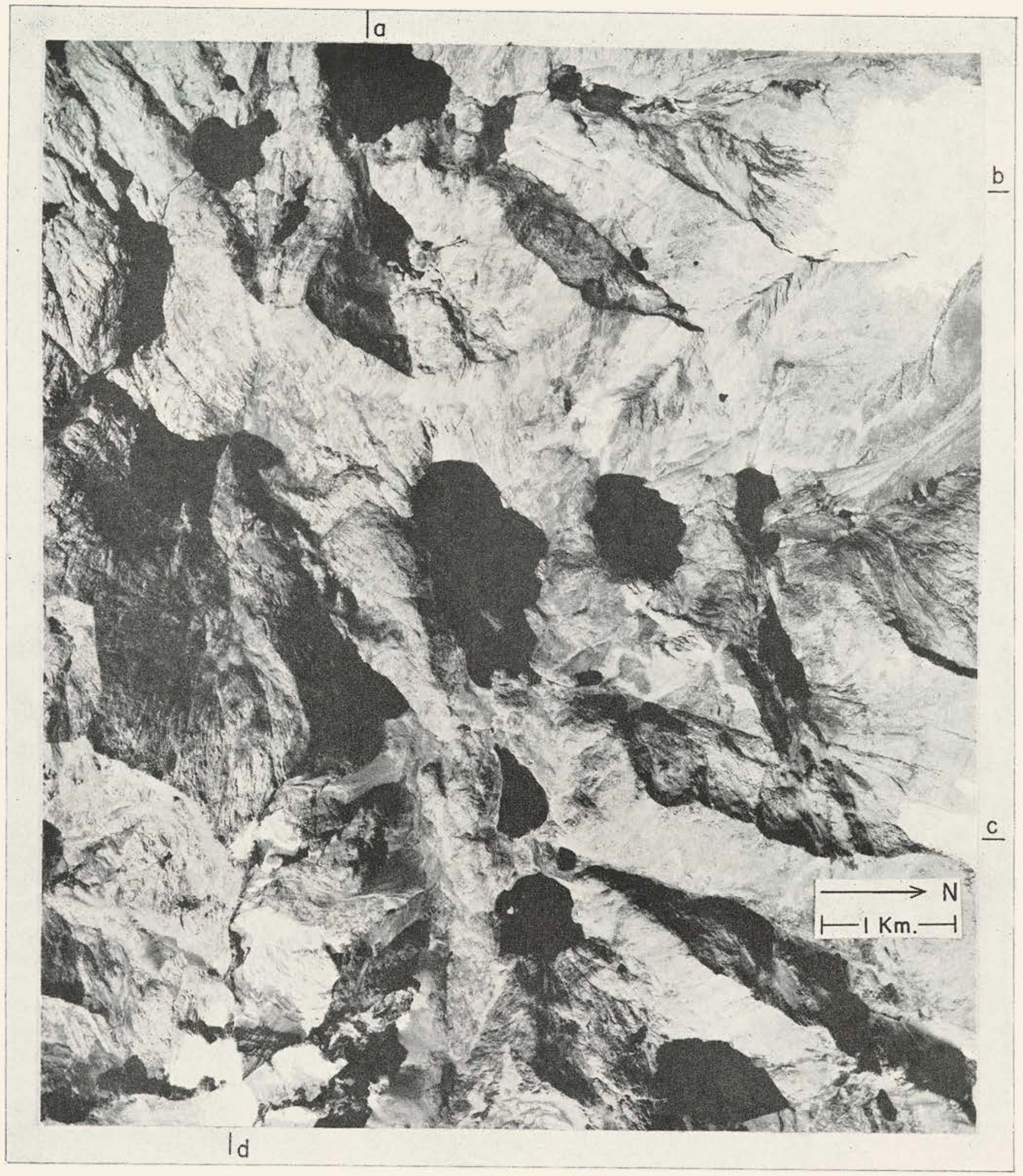

Fig. 3.-Ejemplo de un paisaje dominado por la presencia de ollas glaciales excavadas por el hielo que cubría la región durante estados pre-bolivarianos. Después de retirarse el hielo, se llenaron de agua las ollas glaciales y resultaron los lagcs glaciales, el más grande de los cuales es el Lago Naboba, en el centro, y el Lago del Mamo que apenas entra en la fotografía en el margen superior (a). Los glaciares b, c y d descienden respectivamente del Pico Santander, de la cuchilla al este de los Picos Bolívar-Colón y de los nevados Guardián-Tairona (compárese la fotografía con el mapa glaciológico). 


\section{Las morrenas modernus.}

Alrededor de los glaciares actuales, pero en ge neral separada de ellos, se encuentra una zona de morrenas. Ellas forman un sistema de guirnaldas cuyas partes, cóncavas hacia arriba, indican un mayor avance del hielo en estos sitios. A veces se doblan las guirnaldas en forma de $\mathrm{U}$ lo que indica que una lengua glacial genuina las formó. En ciertos casos se nota una sola morrena individual, pero en general se presentan varias, una tras otra, que corresponden a varias posiciones sucesivas del hielo. Se trata especialmente de morrenas terminales: frontales y laterales. Aunque en los lados cóncavos de las morrenas, es decir en el lado que mira hacia el hielo, hay morrenas de fondo de menor extensión, la zona entre el límite actual de hielos y las morrenas están desprovistas de apreciables depósitos glaciales. Parece entoces que el retroceso de los glaciares fue bastante rápido. La zona en cuestión, entre las morrenas y los actuales glaciares, está conspícuamente desnuda, y en ella la vegetación escasamente se ha arraigado. Zonas semejantes son conocidas y las he observado en los Alpes, donde se las conecta con el retiro del hielo desde un cierto máximo de extensión hasta la posición actual. El máximo más notorio en los Alpes fue el del año 1850 y la zona de escasa vegetación que rodea los glaciares tiene un límite exterior, a cierta distancia del hielo, que se llama "línea de 1850". El conocido profesor austríaco $R$. von Klebelsberg menciona en su manual "Handbuch der Gletscherkunde und Glazialgeologie" tres máximos en el tiempo histórico: los de los años 1600, 1820 y 1850. Hay además, indicios de que antes de 1600, los glaciares estaban bastantes más retirados que hoy en día (antiguas minas de oro debajo de los actuales glaciares). Durante los mencionados máximos de glaciación, los glaciares de valle, o lenguas glaciales, estaban en los Alpes, varios kilómetros más abajo que hoy día, alcanzando una diferencia vertical de 100 a 200 metros con referencia al nivel actual de los glaciares, según von Klebelsberg. Esto es comparable con las circunstancias en la Sierra Nevada de Santa Marta, especialmente si tomamos en consideración el tipo de los glaciares de que se trata aquí La distancia entre el hielo actual y las morrenas más elevadas es en general menor de 500 metros $^{4}$ cuando las guirnaldas no son extremadamente cóncavas, pero se puede aumentar considerablemente, a más de un kilómetro, en los sitios de pronunciadas lenguas glaciales. Considero entonces que el primer grupo de morrenas terminales alrededor de los actuales glaciares, o parte de ellas, es reciente o sea del tiempo histórico. Como están agrupados particualrmente alrededor de las sierras cuya cima dominante es el Pico Simón Bolívar, me referiré a estas morrenas en conjunto como morrenas del Estado Bolivariano. De esta manera obtenemos un nombre provisional para indi-

4 En proyección horizontal car un grupo de morrenas contiguas, nombre general que nos servirá hasta que podamos diferenciar y establecer mejor la cronología de las unidades de que se compone este grupo.

\section{$\mathrm{V}$. Vestigios de glaciaciones prebolivarianas.}

Al lado de la glaciación histórica, la región de la Sierra Nevada de Santa Marta ofrece además claros vestigios de glaciaciones anteriores a las del estado bolivariano.

Esto se manifiesta en particular por la labor del hielo como modelador del paisaje, a saber: la erosión que se caracteriza por formarse planos cepillados, ollas glaciales, lagos glaciales y la acumulación glacial que origina varias clases de morrenas.

Planos cepillados.

La erosión producida en la Sierra Nevada por los glaciares puede conducir a dos tipos de topografía bien distintos entre sí, la topografía de los planos cepillados y la de las ollas glaciales. En el primer tipo se forman superficies planas e inclinadas que resultan a causa del descenso de los glaciares por las pendientes. El tamaño de los planos cepillados puede variar de unos cien metros hasta varios kilómetros de ancho y de largo. No fueron casquetes de lice confinuos que los formaron sino corrientes de hielo divididas en varias unidades cada cual con su propia pendiente, propio aporte de hielo y propia velocidad. En estas condiciones es obvio que el hielo en el límite de dos unidades glaciales tenga menos poder erosivo que en la parte central de la unidad en movimiento y en consecuencia se desarrollaron paredes divisorias entre las diferentes partes de un casquete de hielo. Estas se presentan después de retirarse los glaciares como "costillas" en el terreno, (fig. 14), a veces delgadas con los flancos paralelos, a veces en forma de cuña con los flancos divergentes hacia abajo. Las últimas se forman cuando la topografía tiende a divergir las corrientes de hielo (ver fig. 2).

Los planos cepillados más importantes llevan su símbolo en el mapa glaciológico. Las líneas rojas indican la dirección del movimiento de los hielos.

En el complejo orográfico que se extiende alrededor de los Cerros Hettner (a2), Garavito (b2) y Darío Rozo (b2), los planos cepillados tienen extensiones menores. Los planos más típicos y más grandes se encuentran en una faja del flanco septentrional de los presentes nevados: desde el Cerro Darío Rozo (b2) se extienden al norte del Nevado Parra Lleras, con una prolongación hacia el norte en el cerro Gansser (a3) ; luego dan una vuelta alrededor de los picos Ruiz Wilches y Cristóbal Colón (b4) para continuar en dirección oriental hasta más allá del Cerro Valencia (b6). Además están bien representados en las vertientes de los cerros Guhl (a5) y Grière (b5). También son característicos alrededor del Nevado Ramírez (c5) y en las vertientes al oeste de una línea Nevado Parra Lleras-Cerro Karsten (c3). Al con- 
trario, no están bien formados y pasan gradualmente a la topografía de ollas glaciales al sur de los nevados, al norte del Cerro Arias (d3), al sur-oeste del Cerro Pérez Arbeláez (d3-4) y en las euchillas entre el último y el Pico Tairona.

Ollas glaciales, circos $y$ cols.

El segundo tipo de erosión glacial forma un gran número de concavidades en la roca firme que llamaremos "ollas glaciales" 5 .

Las ollas más grandes y alargadas pueden tener varios kilómetros de largo y un kilómetro de ancho. En general tienen un diámetro de 500 metros a $\mathrm{m}$ kilómetro. Se forman por la labor excavadora de las corrientes de hielo y no deben confundirse con los circos glaciales. Los últimos son unidades más grandes, también medio cóncavas, pero rodeadas por crestas puntiagudas que separan un circo de otro. Estas crestas eran en gran parte desnudas, es decir no cubiertas por el hielo, y la forma angulosa es el resultado de la erosión (por descascaramiento) a causa de las fuertes diferencias de temperatura entre el día y la noche (fig. 15). La localización de los circos puede deducirse a base de las cuchillas divisorias que están indicadas en el mapa. En algunos circos importantes puede observarse el fenómeno de que la erosión del hielo y neviza acumulados en ellos ha avanzado en tal manera hacia arriba, que la cresta divisioria entre dos circos desapareció localmente. Se formó en estas partes una depresión en la cresta, o paso, que se suele llamar "col". Los dos "cols" más importantes los hay elltre los circos superiores de las cuencas de los ríos Cataca-Donachuí, y entre los de los ríos CatacaGuatapurí. El último col que se extiende (perpendicularmente) entre la cresta del Pico Ojeda por un lado y la del Pico Reina por otro lado, está todavía eubierto por hielo y neviza.

Las ollas glaciales se formaron en la región antiguamente cubierta de los hielos y pueden hallarse varias de ellas en un solo circo. Se presentan en los circos, en las vertientes de cuchillas divisorias, pero especialmente en los antiguos valles de glaciares, donde siguen en rápida sucesión, la una tras la otra. La parte posterior, valle arriba, la forma generalmente una pared bastante más inclinada que el perfil longitudinal del valle. El fondo de la olla glacial, desde el pie de la mencionada pared posterior, puede inclinarse ligeramente hacia abajo, ser horizontal o inclinarse hacia las cabeceras del valle. En el último caso tiene un zócalo de roca firme en la parte anterior de la olla, que se llenará de agua y dará origen a un tipo es-

5 Por analogia con las "marmitas glaciales". Las marmitas (en inglés kettles) se forman en las morrenas donde bloques de hielo enterrados en el deposito glacial al derretirse dejan depresiones. Ver R. F. Flint: "Glacial Geology and the Pleistocene Epoch", Wiley and Sons, New York, IV ed., 1953, fig. 41, p. 149, y también A. Holmes: "Geología Fisica", traducción de Rafael Candel y Joaquina de Candel, Barcelona, 1952, pp. 228 y 229 . pecial de lago glacial. En los primeros dos casos el valle muestra un perfil de peldaños.

El paisaje más típico lo forman las ollas glaciales con zócalos, por llenarse éllas de agua (fig. 3 ).

La razón de formarse en un área uma topografía de planos cepillados y en otra una de ollas glaciales consiste, a mi parecer, en el carácter de las rocas que afloran. Si ellas son homogéneas, por ejemplo granitos, eventualmente neisificados, entonces resulta una topografía de planos cepillados. Rocas heterogéneas, como por ejemplo alternaciones de neises compactos con esquistos más blandos, son los terrenos adecuados para la formación de las ollas. La acción excavadora del hielo será especialmente efectiva en aquellas zonas que están fuertemente plegadas, falladas o fracturadas, como lo muestra esquemáticamente la fig. 4.
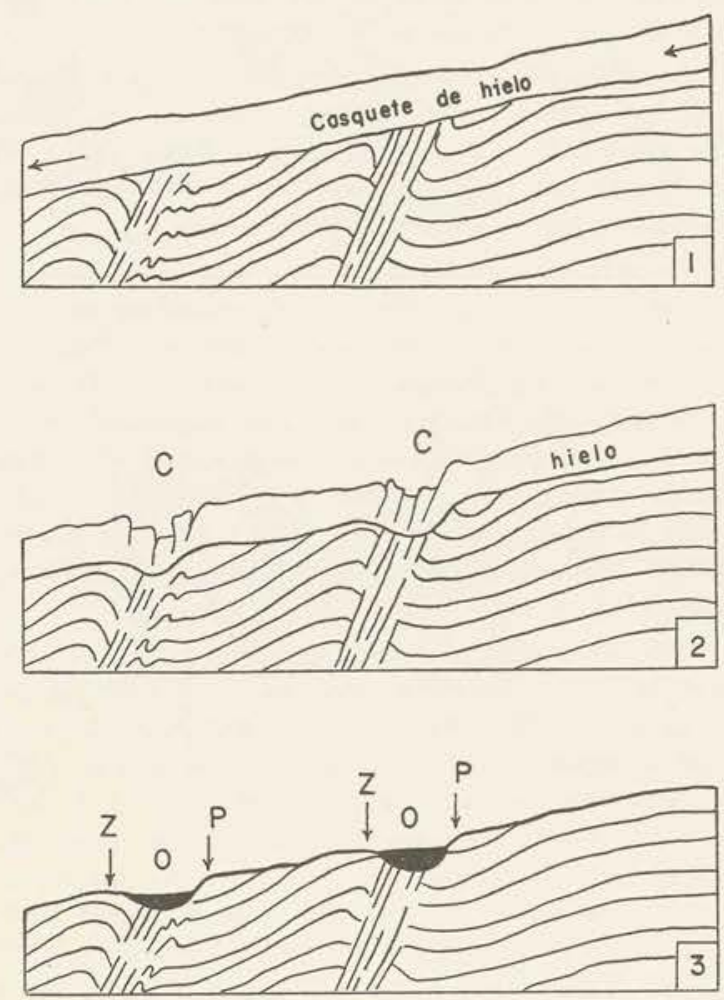

Fig. 4.-Diagramas para explicar la formación de las ollas glaciales.

$\mathrm{C}=$ Cascada de hielo, $\mathrm{O}=\mathrm{O}$ lla glacial, $\mathrm{P}=$ Pared empinada, $\mathrm{Z}=\mathbf{Z}$ ćcalo.

El mapa glaciológico indica las ollas glaciales con un símbolo morfológico especial en forma de herradura.

\section{Lagos glaciales.}

La región con vestigios de glaciación todavía no destruídos por la erosión cuenta con bastantes lagos y laguitos glaciales. Los más grandes son el Lago Naboba, el Lago del Mamo y el lago grande y alongado al norte del C. Pérez Arbeláez. Un gran número de ellos se formó por llenarse de agua las ollas glaciales. En este caso pueden tener un zócalo de roca fija o puede haberse cubierto el zócalo localmente con 
material de morrena. Los lagos de este último tipo no están completamente rodeados de roca firme. Otros lagos se originaron en los valles por la acción bloqueadora de las morrenas terminales de antiguos glaciares.

\section{El grupo die morrenas prebolivarianas, o sea el Estado Mamancanaca.}

Bastante más abajo de las morrenas bolivarianas se encuentra otro complejo de morrenas, una tras otra, zona mucho más amplia que la bolivariana. Debido a que comprende niveles más bajos, esta etapa de glaciación se extiende sobre una región mucho más vasta. Forma guirnaldas alrededor de las unidades orográficas más importantes, y en los valles se presentan morrenas bien desarrolladas en forma de lengua que indican que antes había suficientes circos llenos de abundante neviza que abastecían los glaciares de valle.

Importantes acumulaciones de material glacial las hay en el complejo montañoso de los cerros Hettner (a2), Garavito y Darío Rozo (b2); las morrenas más desarrolladas están situadas en la vertiente oriental del Cerro Garavito.

La faja más ancha de morrenas se encuentra alrededor de los promontorios que descienden hacia el sur de los nevados Ruiz Erazo, Simons, Cristóbal Colón - Bolívar - Vespucio y el Guardián - Tairona. Allí serpentea alrededor de los promotorios, de los cuales forman parte los cerros Karsten (c3), Marmillod (c3), Pérez Arbeláez (d4) y Laverde (c/d5), para luego continuar hacia el sur $\mathrm{y}$ suroeste alrededor del Reclus (d5), Cabot, Arjona (d5) y Kraus (d4).

Morrenas más o menos extendidas en la parte sur del mapa, se encuentran en los flancos de pequeñas unidades orográficas con antiguas glaciaciomes independientes como los cerros Arias (d3), Acosta (d3),Stutzer (e4) Grosse (e4) y Cuenet $(e 4 / 5)$. Varias de apreciable tamaño se presentan en la vertiente meridional de la cuchilla Rodríguez (c6) y en ambos flancos del cerro Valencia (b6). De menor extensión son los restos de morrenas del Cerro Grière (b5) y los que están situados entre P. Ruiz Wilches (b4) y C. Gansser (a3).

Morrenas de fondo, interrumpidas por varias morrenas terminales situadas a diferentes alturas, pueden observarse en los valles más importantes recorridos por el río Tucurinca (c2, ver b3), el río entre C. Marmillod y C. Karsten (c3), el río Cataca (c4, ver d3), el Mamancanaca (d4), el Donachuí (d6, ver c5/6) y el Guatapurí (c6). Son estas las cuencas por las que bajaban los glaciares de valle propiamente dichos. Es probable que parte de estas mencionadas morrenas de valle pertenezcan todavía al estado bolivariano.

En el conjunto de las morrenas terminales se pueden ver todas las transiciones entre guirnaldas apenas cóncavas hasta los tipos de morrenas de lengua en forma de $\mathrm{U}$ comprimida.
Las cabeceras del río Mamancanaca se destacan especialmente por una característica confluencia de varias morrenas de valle que convergen hacia este río desde el SE, E, NE y $\mathrm{N}$ (esquina NE de d4, ver además a fig. 5). Por esta peculiaridad, he decidido referirme a estas morrenas como las del Estado Mamancanaca. Más claramente que en las prebolivarianas se distinguen varias fases de retroceso en las morrenas del Mamancanaca. Considerando además su extensión mucho mayor y su considerable espesor y volumen, merecen más bien el nombre del Gran Estado de Mamancanaca. Es obvio que la extensión de los hielos durante el Gran Estadlo Mamancanaca causó la erosión glacial de la zona entre las mencionadas morrenas y las del estado bolivariano.

Las morrenas terminales del período Mamancanaca pueden variar bastante de altitud; la mayor parte se encuentra entre los 3.500 y 4.000 metros, una altitud media de 3.700 y 3.800 es muy común. Algunas están más arriba de $\operatorname{los} 4.000 \mathrm{~m}$; la altitud más baja a la cual desciende la mayor de las antiguas lenguas glaciales del río Mamancanaca debe ser $3.450 \mathrm{~m}$, aproximadamente. Estas variaciones de altura están directamente conectadas con la extensión y el espesor de los easquetes de hielo. Alrededor de campos extensos de hielo espeso, las lenguas glaciares obviamente podían descender considerablemente más que las provenientes de casquetes pequeños $\mathrm{y}$ de reducido espesor.

\section{Indicios de una glaciación premancanaca. Estado Aduriameina.}

El terreno situado abajo de las morrenas Mamancanaca que rodean los cerros Kraus-Arjona (d4-d5), Stutzer (e4), Grosse (e4) y Cuenet (e 4/5) muestra vestigios de una glaciación más antigua. De norte a sur este terreno se extiende desde la vertiente sureste del río Mamancanaca hasta las cabeceras del río Cuturataca, cruzando la divisoria C. StutzerC. Mamón (o Chu-Chu, e5). Del E al W continúa del mismo C. Mamón hasta unos pocos kilómetros al sur del C. Acosta.

Esta región contiene extensas, pero no muy gruesas, morrenas de fondo recubiertas de nuevo en muchos sitios por depósitos é́licos. EI material de estos depósitos, subaéreos a mi parecer, proviene de las morrenas del Gran Estado Mamancanaca. Una vez formadas estas morrenas, el viento llevó el material más fino y lo depositó en los alrededores más retirados. Los depósitos é́licos requieren un clima frío y seco, y generalmente se les considera formados durante los tiempos glaciales y no durante los interglaciales. Parece entonces que se formaron durante la máxima extensión de los hielos del Gran Estado Mamancanaca y no después. La distribución de los sedimentos é́licos parece apoyar este punto de vista porque se encuentran abajo de las morrenas del Mamancanaca y no más arriba en terreno denudado por la erosión glacial, 
donde ciertamente debieran también estar presentes si se supone que fueron formados después del retiro de los hielos mamancanaquenses. Los depósitos é́licos mamancanaquenses son en todo caso más modernos que las morrenas de fondo que ellos cubren, y esta condición hace que las últimas no sean bien accesibles al estudio. La posición topográfica más baja de estas morrenas, en relación con las mamancanaquenses, obliga a concluir que son más viejas. Pero existe también otro indicio para argüir que se trata en esta región de una topografía más antigua que la del Estado Mamancanaca. En las cabeceras del río Aduriameina existe un valle en forma de una $U$ abierta, vestigio de un antiguo valle glacial que principia al norte del Cerro Cuenet y que desciende hacia el occidente. Al norte del Cerro Stutzer, en vez de bajar hacia el río Mamancanaca, continúa en dirección W o WSW para terminar a una distancia de unos $3 \mathrm{~km}$ al sur del C. Acosta. Tiene dos interrupciones (al norte del Cerro Stutzer y al sureste del Cerro Acosta) que se pueden explicar por los efectos destructores de la erosión más moderna. No obstante esto, se trata de un solo valle glacial, largo $\mathrm{y}$ antiguo. Hacia arriba, este valle no continúa porque las morrenas que descienden de los dos complejos Arjona-Kraus por un lado y las del Cerro Cuenet por otro, obstruyen su continuación hacia arriba. Estas morrenas (mamancanaquenses) son entonces más modernas y el discutido valle glacial debe por consiguiente ser premamancanaquense. Es de interés anotar que el antiguo río que descendía por este valle glacial fue decapitado dos veces por corrientes perpendiculares a la direceión del valle. Una decapitación se puede observar al SE del Cerro Acosta producida por la quebrada que baja hacia el SE para unirse con el río Cuturataca; la otra está al norte del C. Stutzer. La parte del río Aduriameina al oriente de esta localidad es el viejo curso, en cambio la parte que desciende hacia el NW en el R. Mamancanaca es causa de la decapitación más moderna. Esta decapitación debe haberse producido durante una fase de erosión entre los estados glaciales de Mamancanaca y el Premamancanaca (o Aduriameina).

\section{Las diferentes glaciaciones de la Sierra Nevada}

Mencionamos más arriba el hecho de que en la Sierra Nevada de Santa Marta pueden distinguirse tres estados de glaciaciones: el bolivariano, el Mamancanaca y el premamancanaca que podemos llamar más explicítamente Aduriameina. Los primeros dos estados se pueden subdividir en varias fases, reflejadas en las morrenas, cuando el hielo avanzó algo y lnego retrocedió.

La misma posición de las morrenas terminales nos permite reconstruir la extensión aproximada de los hielos durante estos estados. Estos se han hecho en las figuras 7 y 8 ; para facilitar comparaciones presentamos en la misma escala la extensión de los glaciares actuales (fig. 6). Indicamos también las cuchillas divisorias entre circos actuales y antiguos, las direcciones de las corrientes de hielo y los más importantes valles glaciales en la figura 8 .

Las superficies cubiertas por las máximas glaciaciones y sus proporciones relativas están reunidas en la siguiente tabla:

\begin{tabular}{lrcl} 
Glaciaciones & Superficie ${ }^{6}$ & $\begin{array}{c}\text { Proporciones com- } \\
\text { parativas }\end{array}$ \\
Reciente & \multicolumn{4}{c}{ Sm $^{2}$} & 1 & 0,37 \\
Bolívar & $105 \mathrm{~km}^{2}$ & 2,7 & 1 \\
Mamancanaca & $856 \mathrm{~km}^{2}$ & 22 & 8,15 \\
Aduriameina & $?$ & &
\end{tabular}

Acerca de la eronología de los estados de glaciación, ningún dato exacto puede darse por el momento y sólo podemos presentar algunas consideraciones especulativas. Parece bastante seguro que las morrenas bolivarianas son modernas.

Las del estado Mamancanaca ya podrían pertenecer al Pleistoceno por su magnitud y grande extensión. Los depósitos élicos alrededor de ellos también podrían indicar una edad pleistocena, por su conexión con condiciones climatológicas más rigurosas que las de los tiempos modernos. La fuerte erosión glacial del terreno cansada por los hielos mamancanaquenses podría también apoyar esta conclusión. El recubrimiento sumamente escaso con materiales glaciales de la zona entre las morrenas Mamancanaca y las bolivarianas indica un rápido retiro de los glaciares sobre una apreciable distancia. Es muy probable que los glaciares desaparecieron completamente entre los estados MamancanacaBolívar o que se redujeron a una extensión menor que los glaciares actuales. Según estas sugerencias, sería justificado suponer que las morrenas del período Mamancanaca son pleistocenas, es decir, würmianas, para hablar en términos internacionales.

Por otra parte, se conocen en Europa y Norteamérica varios importantes estados de glaciación después del-último Würmiano propiamente dicho, estados que varios autores en su conjunto llamaron "La glaciación final", aunque es muy dudoso que se trate de un verdadero período de glaciación comparable a los pleistocenos (Würm, Riss, etc.). En todo caso, prácticamente no habrá campo para colocar todos estos estados en retroceso en el conjunto de fenómenos de glaciación de la Sierra Nevada de Santa Marta, si se atribuye una edad würmiana (es decir pleistocena) al período de Mamancanaca. Por esta razón me parece más indicado paralelizar por el momento el Mamancanaca con los estados más antiguos de las "glaciaciones finales".

Los restos de una glaciación propiamente pleistocena, o würmiana, deberían buscarse entonces más abajo de las morrenas mamancanaquenses. Según esta interpretación, las morrenas würmianas ha-

\footnotetext{
6 En proyeccion sobre el plano horizontal.
} 


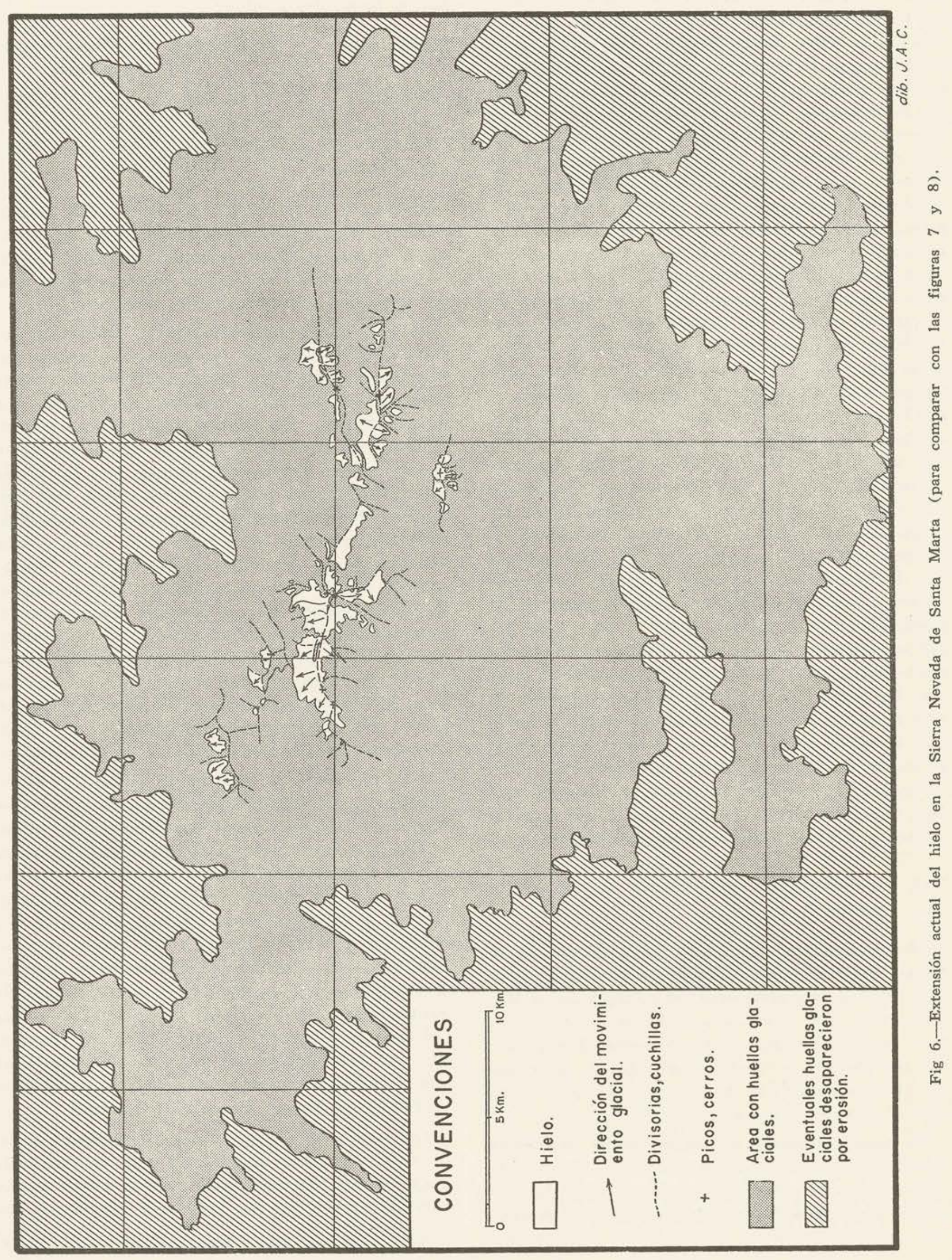




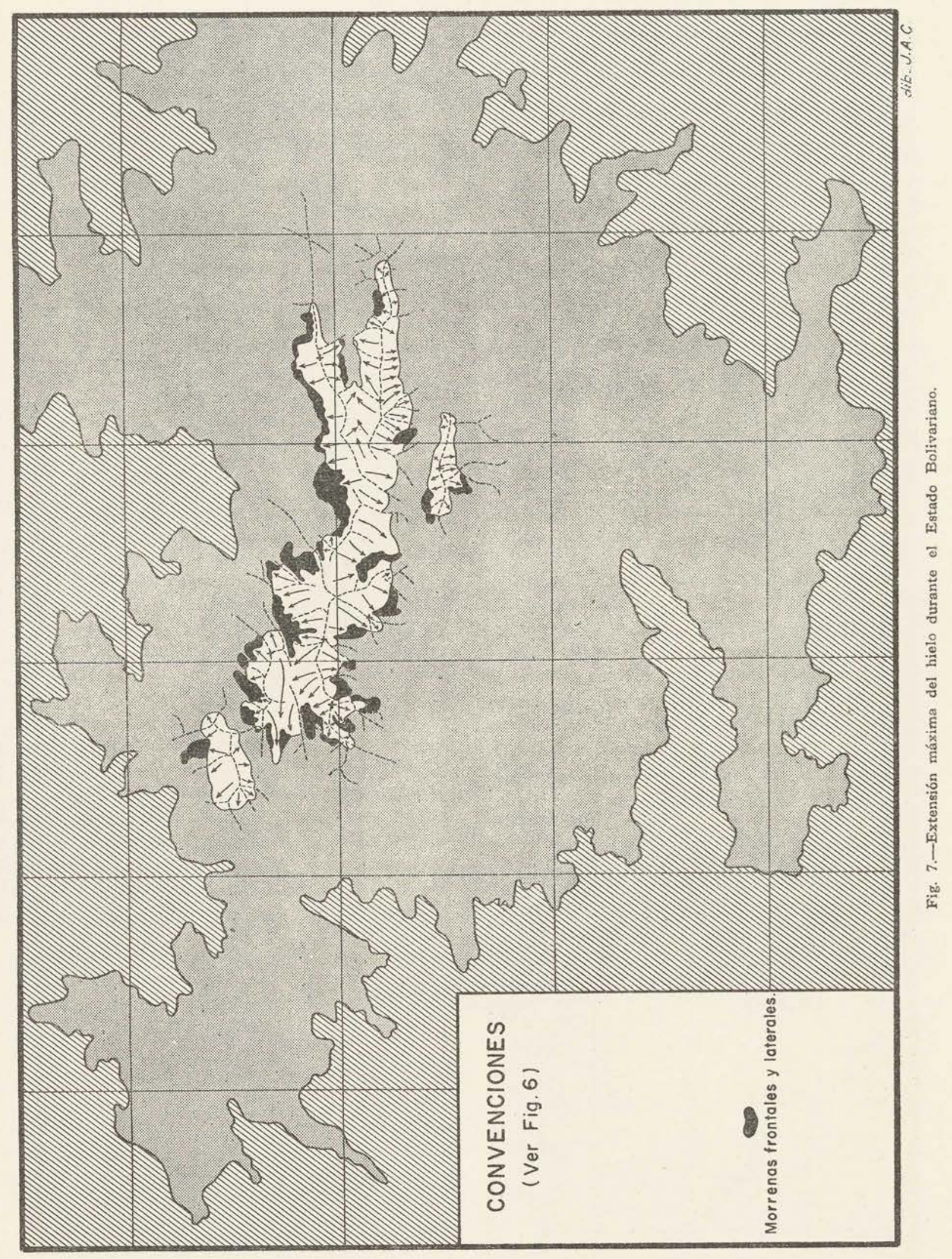




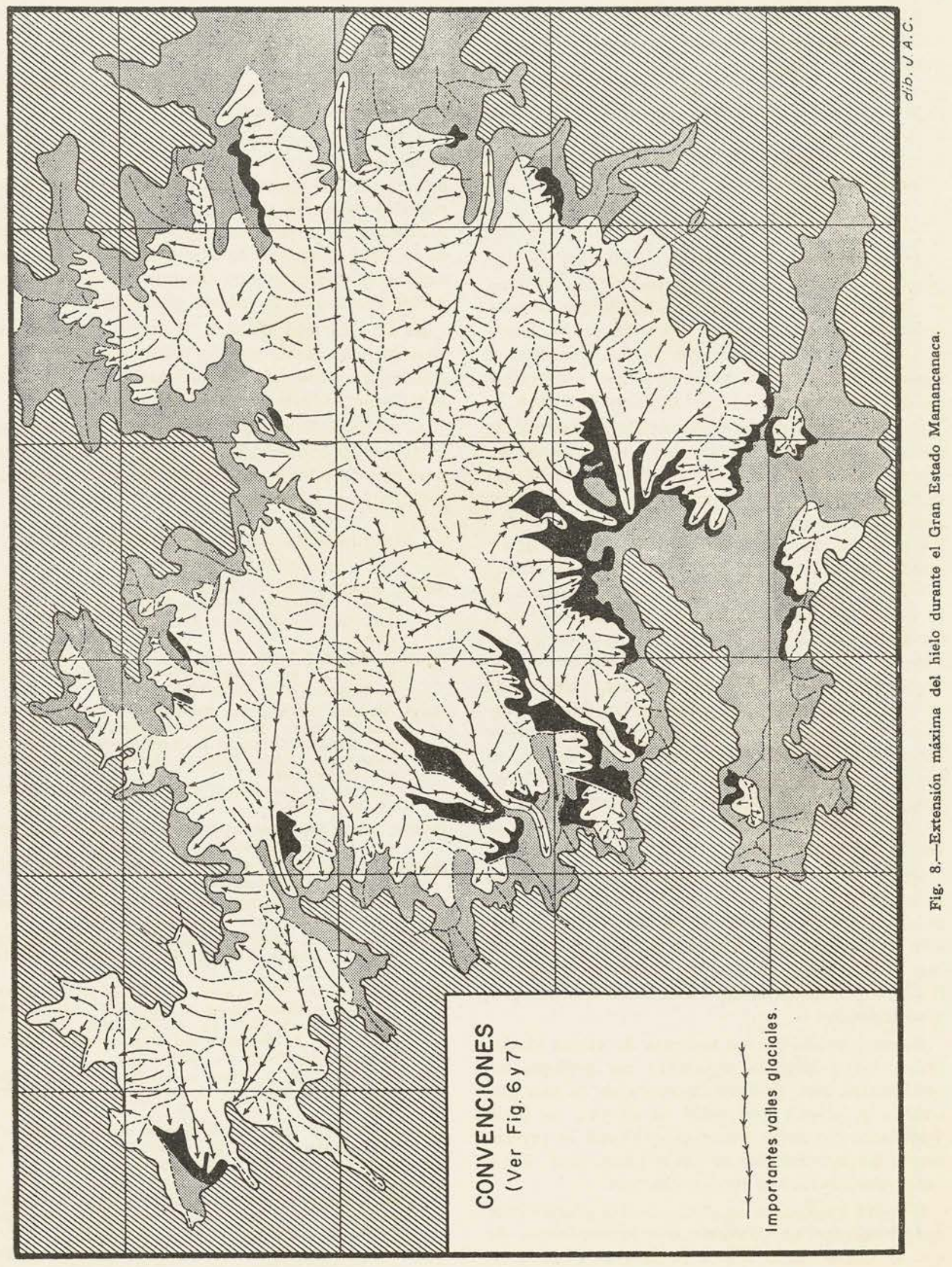


brían sido destruidas en gran parte. Los pocos testigos glaciales todavía conservados del período Aduriameina, podrían entonces corresponder al Würmiano.

Como lo comprenderá el lector, el presente estudio es sólo una orientación general para reconocer la extensión y la intensidad de los vestigios glaciales, y que deben seguir estudios más detallados los cuales requerirán sin duda varios años de intensa labor. Tal empeño sólo tendrá éxito si se dispone de mapas topográficos detallados y provistos de curvas de nivel.

VIII. Nota sobre un reciente estudio de A. Gansser.

Al terminarse el manuscrito recibimos un importante artículo por A. Gansser: "Ein Beitrag zur Geologie und Petrographie der Sierra Nevada de Santa Marta", incluído en la Bibliografía. Gansser ilustra profusamente con cartas, secciones y figuras la descripción de la geología y petrografía de la región cubierta por el mapa de Cabot.

Con referencia al presente estudio es interesante observar que dicho artículo tiene un capítulo dedicado a las glaciaciones de la Sierra Nevada, con un mapa glaciol6gico. También Gansser distingue tres estados de glaciación, los cuales corresponden a los nuestros. Es una feliz coincidencia que su segundo estado lo llama igualmente "Mamancanaca" por la abundancia de morrenas que caracterizan es. te estado en las cabeceras del río Mamancanaca. Su último estado, que corresponde a nuestro "bolivariano", lo llama glaciación reciente hasta subreciente. Inciertos restos de morrenas, vestigios de su glaciación más vieja, que corresponden probablemente a nuestro "Estado Aduriameina", fueron encontrados por Gansser en el río Cuturataca, al sur y sureste del Cerro Stutzer (e4). Observ6 también arriba de los 3.000 metros el caracter glacial de varios valles, que él conecta también con el estado más antiguo.

Abajo de los 3.000 metros comprobó un aumento de las gradientes de los ríos y es aparente que esta peculiaridad corresponde al límite de intensa erosión fluvial que está ascendiendo desde el nivel del mar (línea roja en nuestro mapa glaciológico), límite, cuya altura media es de 3.000 metros aproximadamente.

Gansser opina que no se trata de varias glaciaciones independientes, separadas por periodos interglaciales, sino de fases de retroceso de una glaciación principal, que sería la altima, es decir würmiana. No hallo restos que indican la presencia de glaciaciones más antiguas (Riss, etc.). Estos resultados concardan con los nuestros.

Gansser explica la ausencia de las glaciaciones pre-würmianas en Colombia con la suposición de que el macizo de la Sierra Nevada de S. M. y en general las otras cordilleras colombianas no fueron levantadas hasta el nivel de la neviza sino después de la penaltima glaciacion pleistocena (Riss).
Esta idea nos parece de importancia trascendental y su eventual comprobación en el futuro tendrá. consecuencias de mucho alcance para la tectónica y la geología cuaternaria de estas latitudes tropicales.

\section{Agradecimientos.}

- El autor quiere expresar sus agradecimientos a las siguientes personas:

A Erwin Kraus por prestar su entusiasta colaboracion que permitio completar la nomenclatura del mapa; por las descripciones que nos hizo de las regiones visitadas por él y por haber puesto generosamente a nuestra disposición una selección de su extenso archivo de excelentes fotografias, del cual escogimos once para ilustrar este artículo con varios fenomenos de interés glaciologico.

Al Dr. Enrique Hubach, Director del Instituto Geol6gico Nacional, por las numerosas sugerencias que mejoraron la redacción del artículo y por haber autorizado su publicación en esta revista.

A Antonio Tomic por la estrecha y constante colaboracion prestada en todas las fases de la preparacion de este estudio, tanto del texto, como de las figuras y del mapa.

\section{BIBLIOGRAFIA DE LA SIERRA NEVADA DE SANTA MARTA}

Acosta, Joaquín, "Sur le Géologie de la Sierra Taírona (Nouv. Grenade)", (carta a Elie de Baumont) Bull. Soc. Geol. France.; T. 9, Ser. 2, pp 390-399, Plancha, 1851.

Acosta, Joaquín, "Sur la Sierra Nevada de Sainte Marthe, formée par les terrains primitifs" Bull. Soc. Géol. de France. Vol, 9, París 1852.

Bergt, Walter, "Beitrag zur Petrographie der Sierra Nevada de Santa Marta und der Slerra de Perijá in der Republik Columbia in Suedamerika". Inaugural Dissertation (Univ. Leipzig), Alered Hoelder, Wien, pp. $271-386,1888$,

Tambiẹn en Tschermaks min. u. petr. Mitt., $10, \mathrm{~N}$ ? 4-5, Wien. 1888 .

Brettes, Joseph de, *Chez les Indiens du Nord de la Colombie", Le Tour du Monde, vol. IV, 1898.

Brettes, Joseph de, "Les Indiens Arhuaques-Kaggabas", Bull. de la Soc. d'Antropol., París, 1902.

Cabot, Thomas D., "Mountains of the Caribbean", Appalachia, Boston, Vol. 18 pp. 17-22, 1930.

Cabot, Thomas D., "Slerra Nevada de Santa Marta", Appalachia, Boston, Vol. 22, No 87, Jun., pp. 309-314, 1939.

Cabot Thomas D., "The Cabot Expedition to the Sierra Nevada de Santa Marta of Colombia", Geog. Rev., Vol. 29 , N9 4 , Oct., pp. 587-621. Map. by Wood, Waiter A., 2 "Geologic and Physiographic Notes", by Notestein Frank B., 1939.

Caracristi, M. C. F. Z., "Una excursión a la Sierra Nevada", Anal Ing., Vol. 7 , $\mathrm{N}^{\circ} 81$, Sep., pp. 269-271, 1894.

Calderón, Rafael, "La Sierra Nevada de Santa Marta", Corr, Aldeas, Ser, 1, No 3; Ago.; pp. 28-42; No 4; Ago., pp. 56-59; No 5, Ago, pp. 70-73, 1887.

Cornette, A., "Extraits de differrentes lettres adressées a M. Deshayes par le pere Cornette, de la compagnie de Jesús", Bull. Soc. Géol. France, T 9, Deuxieme Série, pp. 509-560.

Cuénet, G. H., "Excursión del Dr. G. H. Cuénet a la Sierr2 Nevada de Santa Marta". Vínculo, No 34, Marzo 1951 (Organo de la Cía. Colombiana de Petróleo FI Cóndor y de la Cía. de Petróleo Shell de Colombia. Excursión 21 enero - 7 febrero 1951: Erwin Kraus y Raymond Grière; Guardián, Simón Bolívar). 
Flye, Oriando L., "Mis impresiones de la Sierra Nevada de Santa Marta", Pan. N9 3, Oct., pp. 27-31 (tomado de "El IEstado", No 2548), 1935.

Gansser, August, "Ein Beitrag zur Geologie und Petrographie der Sierra Nevada de Santa Marta", Bull. suisse de Minéralogie et Pétrographie, Bd 35, H 2, 1955 , pp. $209-279$.

Gerth, Heinrich, "Der Geologische Bau der suedamerikanischen Kordillere", Gebrueder "Borntraeger, Berlín, 1955. La Sierra Nevada de S. M. en pág. 153, $\mathrm{n}_{0}$ se refiere a las glaciaciones.

Gómez Picón, Rafael, "Visión de la Sierra Tayrona", Bol. Soc. Geog. Colom., Vol. 8, No 2, Dic., pp. 180-184, Fotos, 1948.

Grière, Raymond, "La Sierra Nevada de Santa Marta". Alpinisme, printemps 1952 (179, rue de Pompe, París $\left.\mathrm{XVI}^{\mathrm{e}}\right)$, No 99, pp. 17-24; un mapa con las rutas de Kraus-Cabot, Cuénet-Perret, Cuénet-Grière-Kraus; seis fotografias, bnena historia de todas las excursiones.

Guhl, Ernesto, "La Sierra Nevada de Santa Marta", Rev. Acad. Colom, Cienc. Exact. Fís. Nat. Vol. 8, No 29, Nov., pp 111-122, Planchas, Lámina, 1950.

Hall, H. S., "Sierra Nevada de Santa Marta, Colombia", Can. Alp. Jour. Vol. 31, No 12, Dic., pp 2171-2192, Figs., 1947.

Hettuer, Alfred, "Die Sierra Nevada ron Santa Marta", Peterm, Mitt., Vol. 21, pp 92-97, 1885.

Hubach, Enrique, "La Sierra Nevada de los Taironas", Pan, N9 3, Oct., pp 1-14. Fotos, 1935.

Karsten, Hermann, "Geognostische Bemerkungen ueber die Nordkueste Neu-Granadas, insbesondere ueber die sogenannten Vulkane ron Turbaco und Zamba", Zeitschr. D. Geol. Gesells., Band 4, Heft 3, MayJul., 1952.

Karsten, Hermann, "Ueber die geognostischen Verhaeltnisse des westlichen Columbien, der heutigen Republiken Neu Granada und Ecuador", Lith. u. ged. i.k.k. Hof u. Staatsdruckerei, Wien, 1856.

Kraus, Erwin, "Ascenso a la Sierra Nevada de Santa Marta", Pan, No 31, Jun. 26, 1939.

Oppenheim, Victor, "Geología del Departamento del Magdalena", Compil. de los Estudios geol. offic. en Colombia, Tomo 5, Bogotá, 1942.

Raymond, Edouard, "Informe sobre una misión geológica en los departamentos del Magdalena y Atlántico", Comp. de los Estudios geol. ofic. en Colombia, Tomos 5 y 6 , Bogotá, 1942.

Reclus, Elisée, "Voyaye a la Sierra-Nevada de SainteMarthe", Librairie Hachette et Cie., Paris, 338, 21 gravures, 1 carte. 1881
Rosales José Miguel, "Geografía Económica de la Sierra Nevada de Santa Marta", Bol. Soc. Geogr. Colom. Aก̃o $1, \mathrm{~N}^{Q} 2,1934$.

Schenck, Friedrich von, "Reisen in Antioguia im Jahre 1880". Peterm. Mitt. Band 29, Heft 3, pp 81-93 Heft 6 , pp 213-220; Heft 12, pp 411-453, Maps. 1883.

Seifritz, William, "The Sierra Nevada de Santa Marta An ascent from the North", Geogr. Rev., Vol. 24 No 3 , pp 478-485, 1934.

Seifritz, William, "Die Höhenstufen der Vegetation in der Sierra Nevada de Santa Marta", Bot. Jahrb. Vol. $68,1937$.

Sievers, Wilhelm, "Die Sierra Nevada de Santa Marta", Leipzig, 1887.

Sievers, Wilhelm, "Die Sierra Nevada de Santa Marta und die Sierra de Perijá". Zeitschr. Gesellsch. fuer Erdkunde, Bd 23, Berlín, 1888.

Sievers, Wilhelm, "Erlaenterungen zur geognostischen Karte der Sierra Nevada de Santa Marta", Zeits. Gesell. Erdk. Ber. No 137, Oct., Map. Taf., 1888.

Simons, F. A. A., "Notes on the topography of the Sierra Nevada of Santa Marta, U. S. of Colombia", Royal Geog. Soc. Proc. Vol. 1, Ne 11, Nov., pp 689-723. Mapa, 1881.

Simons, F. A. A., "On the Sierra Nevada de Santa Marta and its Watershed (State of Magdalena, U. S. of Colombia)", Vol. 3, No 12, Dic., pp 705-723, Mapa, 1881.

Taylor, Griffith, "Settlement zones of the Sierra Nevada de Santa Marta, Colombia", Geog. Rev., Vol. 21, $\mathrm{N}^{\circ} 3$, pp 539-558, Figs., 1931.

Thevernin, Ernest, "Proyecto de exploración a la Sierra Nevada de Santa Marta, con fines prácticos", Re vista de Industrias, Bogotá, Vol. 1, No 7 , Die., pP 230-233. Mapa, 1924.

Vinalesa, José de, "La Sierra Nevada de Santa Marta. Etnografía de los Bintukuas", Colombia, A. 1, Nos. 1-2 Ene-Feb., pp 66-76, Fotos, 1944

Vinalesa, José de, "El Indio de la Sierra Nevada", Co. lombia, A. 1, Nos. 3-4 Mar-Abr., pp 76-86, Fotos, 1944 .

White, Robert Blake, "Brief notes on the Glacial Phenomena of Colombia (south America)", Scott. Geog. Mag., Vol. 15, No 9, Sep., pp. 470-479, Fotos, 1899.

Wollaston, A. F. R., "The Sierra Nevada de Santa Marta" Geog. Journ., Vol. 66, No 2, Ago., pp 97-111, 1925.

Wood, WaIter A., "Mapping the Sierra Nevada de Santa Marta; The Work of the Cabot Colombian Expedition", Geog. Rev., Vol. 31, pp. 632-639, Mapa, 1941. 